\title{
HERMITE-HADAMARD TYPE INEQUALITIES FOR QUASI-CONVEX FUNCTIONS VIA KATUGAMPOLA FRACTIONAL INTEGRALS
}

\author{
ERHAN SET*, ILKER MUMCU \\ Department of Mathematics, Faculty of Science and Arts, Ordu University, Ordu, Turkey \\ ${ }^{*}$ Corresponding author: erhanset@yahoo.com
}

\begin{abstract}
The paper deals with quasi-convex functions, Katugampola fractional integrals and HermiteHadamard type integral inequalities. The main idea of this paper is to present new Hermite-Hadamard type inequalities for quasi-convex functions using Katugampola fractional integrals, Hölder inequality and the identities in the literature.
\end{abstract}

\section{INTRODUCTION}

A function $f: I \subseteq \mathbb{R} \rightarrow \mathbb{R}$ is said to be convex if the inequality

$$
f(\lambda u+(1-\lambda) v) \leq \lambda f(u)+(1-\lambda) f(v)
$$

holds for all $u, v \in I$ and $\lambda \in[0,1]$. This definition has been used in the following inequality that is called Hermite-Hadamard inequality:

Let $f: I \subseteq \mathbb{R} \rightarrow \mathbb{R}$ be a convex function and $a, b \in I$ with $a<b$, then

$$
f\left(\frac{a+b}{2}\right) \leq \frac{1}{b-a} \int_{a}^{b} f(x) d x \leq \frac{f(a)+f(b)}{2} .
$$

This inequality has attracted many mathematicians. Especially, in the last three decades, numerous generalizations, variants, and extensions of this inequality have been presented (see, e.g., $[1,3,13,14,20]$ and the references cited therein).

Received 2017-10-26; accepted 2018-01-03; published 2018-07-02.

2010 Mathematics Subject Classification. 26A33, 26D10, 33B20.

Key words and phrases. Hermite-Hadamard inequality; Riemann-Liouville fractional integrals; Katugampola fractional integrals.

(C) 2018 Authors retain the copyrights of their papers, and all open access articles are distributed under the terms of the Creative Commons Attribution License. 
The notion of quasi-convex functions generalizes the notion of convex functions. More precisely, a function $f:[a, b] \rightarrow \mathbb{R}$ is said quasi-convex on $[a, b]$ if

$$
f(\lambda u+(1-\lambda) v) \leq \max \{f(x), f(y)\}
$$

for any $x, y \in[a, b]$ and $\lambda \in[0,1]$. Clearly, any convex function is a quasi-convex function. Furthermore, there exist quasi-convex functions which are not convex (see [4]).

Let $f \in L_{1}[a, b]:=L(a, b)$. The Riemann-Liouville integrals $J_{a+}^{\alpha} f$ and $J_{b-}^{\alpha} f$ of order $\alpha \in \mathbb{R}^{+}$are defined, respectively, by

$$
J_{a+}^{\alpha} f(x)=\frac{1}{\Gamma(\alpha)} \int_{a}^{x}(x-t)^{\alpha-1} f(t) d t \quad(x>a)
$$

and

$$
J_{b-}^{\alpha} f(x)=\frac{1}{\Gamma(\alpha)} \int_{x}^{b}(t-x)^{\alpha-1} f(t) d t \quad(x<b)
$$

where $\Gamma$ is the familiar Gamma function (see, e.g., [21, Section 1.1]). It is noted that $J_{a+}^{1} f(x)$ and $J_{b-}^{1} f(x)$ become the usual Riemann integrals.

In the case of $\alpha=1$, the fractional integral reduces to classical integral.

For further results related to Hermite-Hadamard type inequalities involving fractional integrals on can see $[7,11-19]$.

The beta function $B(\alpha, \beta)$ is defined by (see, e.g., [21, Section 1.1] [10, p18])

$$
B(\alpha, \beta)=\left\{\begin{array}{lc}
\int_{0}^{1} t^{\alpha-1}(1-t)^{\beta-1} d t & (\Re(\alpha)>0 ; \Re(\beta)>0) \\
\frac{\Gamma(\alpha) \Gamma(\beta)}{\Gamma(\alpha+\beta)} & \left(\alpha, \beta \in \mathbb{C} \backslash \mathbb{Z}_{0}^{-}\right) .
\end{array}\right.
$$

The hypergeometric function [6]:

$$
{ }_{2} F_{1}(a, b ; c ; z)=\frac{1}{\beta(b, c-b)} \int_{0}^{1} t^{b-1}(1-t)^{c-b-1}(1-z t)^{-a} d t, \quad c>b>0,|z|<1 .
$$

A hypergeometric function can be written using Euler's hypergeometric transformations $(t \rightarrow 1-t)$ in equivalent form:

$$
{ }_{2} F_{1}(a, b ; c ; z)=(1-z)^{-a}{ }_{2} F_{1}\left(a, c-b ; c ; \frac{z}{z-1}\right)
$$

Lemma 1.1. [9] For $0<\alpha \leq 1$ and $0 \leq a<b$, we have

$$
\left|a^{\alpha}-b^{\alpha}\right| \leq(b-a)^{\alpha}
$$

In [11], Sarıkaya et. al. proved a new version of Hermite-Hadamard's inequalities in Riemann-Liouville fractional integral form as follows: 
Theorem 1.1. Let $f:[a, b] \rightarrow \mathbb{R}$ be a positive function with $0 \leq a<b$ and $f \in L_{1}[a, b]$. If $f$ is a convex function on $[a, b]$, then the following inequalities for fractional integrals holds:

$$
f\left(\frac{a+b}{2}\right) \leq \frac{\Gamma(\alpha+1)}{2(b-a)^{\alpha}}\left[J_{a+}^{\alpha} f(b)+J_{b-}^{\alpha} f(a)\right] \leq \frac{f(a)+f(b)}{2}
$$

with $\alpha>0$.

In [8], Özdemir et. al. gave following results for quasi-convex functions via Riemann-Liouville fractional integrals.

Theorem 1.2. Let $f:[a, b] \rightarrow \mathbb{R}$, be a positive function with $0 \leq a<b$ and $f \in L_{1}[a, b]$. If $f$ is a quasi-convex function $[a, b]$, then the following inequality for fractional integrals holds:

$$
\frac{\Gamma(\alpha+1)}{2(b-a)^{\alpha}}\left[J_{a+}^{\alpha} f(b)+J_{b-}^{\alpha} f(a)\right] \leq \max \{f(a), f(b)\}
$$

with $\alpha>0$.

Theorem 1.3. Let $f:[a, b] \rightarrow \mathbb{R}$, be a differentiable mapping on $(a, b)$ with $a<b$. If $\left|f^{\prime}\right|$ is quasi-convex on $[a, b]$ with $\alpha>0$, then the following inequality holds:

$$
\begin{aligned}
& \left|\frac{f(a)+f(b)}{2}-\frac{\Gamma(\alpha+1)}{2(b-a)^{\alpha}}\left[J_{a+}^{\alpha} f(b)+J_{b-}^{\alpha} f(a)\right]\right| \\
\leq & \frac{b-a}{\alpha+1}\left(1-\frac{1}{2^{\alpha}}\right) \max \left\{f^{\prime}(a), f^{\prime}(b)\right\} .
\end{aligned}
$$

Theorem 1.4. Let $f:[a, b] \rightarrow \mathbb{R}$, be a differentiable mapping on $(a, b)$ such that $f^{\prime} \in L_{1}[a, b]$. If $\left|f^{\prime}\right|^{q}$ is quasi-convex on $[a, b]$, and $p>1$, then the following inequality for fractional integrals holds:

$$
\begin{aligned}
& \left|\frac{f(a)+f(b)}{2}-\frac{\Gamma(\alpha+1)}{2(b-a)^{\alpha}}\left[J_{a+}^{\alpha} f(b)+J_{b-}^{\alpha} f(a)\right]\right| \\
\leq & \frac{b-a}{2(\alpha p+1)^{1 / p}}\left(1-\frac{1}{2^{\alpha}}\right)\left(\max \left\{\left|f^{\prime}(a)\right|^{q},\left|f^{\prime}(b)\right|^{q}\right\}\right)^{1 / q} .
\end{aligned}
$$

where $\frac{1}{p}+\frac{1}{q}=1$ and $\alpha \in[0,1]$.

Theorem 1.5. Let $f:[a, b] \rightarrow \mathbb{R}$, be a differentiable mapping on $(a, b)$ such that $f^{\prime} \in L_{1}[a, b]$. If $\left|f^{\prime}\right|^{q}$ is quasi-convex on $[a, b]$, and $q \geq 1$, then the following inequality for fractional integrals holds:

$$
\begin{aligned}
& \left|\frac{f(a)+f(b)}{2}-\frac{\Gamma(\alpha+1)}{2(b-a)^{\alpha}}\left[J_{a+}^{\alpha} f(b)+J_{b-}^{\alpha} f(a)\right]\right| \\
\leq & \frac{b-a}{\alpha+1}\left(1-\frac{1}{2^{\alpha}}\right)\left(\max \left\{\left|f^{\prime}(a)\right|^{q},\left|f^{\prime}(b)\right|^{q}\right\}\right)^{1 / q} .
\end{aligned}
$$

with $\alpha \in[0,1]$.

Katugampola gave a new fractional integral that generalizes the Riemann-Liouville and the Hadamard fractional integrals into a single form. 
Definition 1.1. [5] Let $[a, b] \subset \mathbb{R}$ be a finite interval. Then, the left- and right-side Katugampola fractional integrals of order $(\alpha>0)$ of $f \in X_{c}^{p}(a, b)$ are defined:

$$
{ }^{\rho} \mathcal{I}_{a+}^{\alpha} f(x)=\frac{\rho^{1-\alpha}}{\Gamma(\alpha)} \int_{a}^{x} \frac{t^{\rho-1}}{\left(x^{\rho}-t^{\rho}\right)^{1-\alpha}} f(t) d t
$$

and

$$
{ }^{\rho} \mathcal{I}_{b-}^{\alpha} f(x)=\frac{\rho^{1-\alpha}}{\Gamma(\alpha)} \int_{x}^{b} \frac{t^{\rho-1}}{\left(t^{\rho}-x^{\rho}\right)^{1-\alpha}} f(t) d t
$$

with $a<x<b$ and $\rho>0$, if the integral exist.

Theorem 1.6. [5] Let $\alpha>0$ and $\rho>0$. Then for $x>a$,

1. $\lim _{\rho \rightarrow 1}{ }^{\rho} \mathcal{I}_{a+}^{\alpha} f(x)=J_{a+}^{\alpha} f(x)$,

2. $\lim _{\rho \rightarrow 0^{+}}{ }^{\rho} \mathcal{I}_{a+}^{\alpha} f(x)=H_{a+}^{\alpha} f(x)$.

Similar results also hold for right-sided operators.

In [2], Chen and Katugampola proved the following lemma:

Lemma 1.2. Let $f:\left[a^{\rho}, b^{\rho}\right] \rightarrow \mathbb{R}$ be a differentiable mapping on $\left(a^{\rho}, b^{\rho}\right)$ with $0 \leq a<b$. Then the following equality holds if the fractional integrals exist:

$$
\begin{aligned}
& \frac{f\left(a^{\rho}\right)+f\left(b^{\rho}\right)}{2}-\frac{\alpha \rho^{\alpha} \Gamma(\alpha+1)}{2\left(b^{\rho}-a^{\rho}\right)^{\alpha}}\left[{ }^{\rho} \mathcal{I}_{a+}^{\alpha}(f \circ g)(b)+{ }^{\rho} \mathcal{I}_{b-}^{\alpha}(f \circ g)(a)\right] \\
= & \frac{b^{\rho}-a^{\rho}}{2} \int_{0}^{1}\left[\left(1-t^{\rho}\right)^{\alpha}-t^{\rho \alpha}\right] t^{\rho-1} f^{\prime}\left(t^{\rho} a^{\rho}+\left(1-t^{\rho}\right) b^{\rho}\right) d t
\end{aligned}
$$

where $g(x)=x^{\rho}$.

The main purpose of this paper is to establish Hermite-Hadamard's inequalities for quasi-convex functions via Katugampola fractional integral. We also obtain Hermite-Hadamard type inequalities of these classes functions.

\section{Main Results}

Theorem 2.1. Let $\alpha>0$ and $\rho>0$. Let $f:\left[a^{\rho}, b^{\rho}\right] \rightarrow \mathbb{R}$ be a positive function with $0 \leq a<b$ and $f \in X_{c}^{p}\left(a^{\rho}, b^{\rho}\right)$. If $f$ is a quasi-convex function on $\left[a^{\rho}, b^{\rho}\right]$, then the following inequalities holds:

$$
\frac{\rho^{\alpha} \Gamma(\alpha+1)}{2\left(b^{\rho}-a^{\rho}\right)^{\alpha}}\left[{ }^{\rho} \mathcal{I}_{a+}^{\alpha}(f \circ g)(b)+{ }^{\rho} \mathcal{I}_{b-}^{\alpha}(f \circ g)(a)\right] \leq \max \left\{f\left(a^{\rho}\right), f\left(b^{\rho}\right)\right\}
$$

where $g(x)=x^{\rho}$.

Proof. Since $f$ is quasi-convex function on $\left[a^{\rho}, b^{\rho}\right]$, we get

$$
f\left(t^{\rho} a^{\rho}+\left(1-t^{\rho}\right) b^{\rho}\right) \leq \max \left\{f\left(a^{\rho}\right), f\left(b^{\rho}\right)\right\}
$$


and

$$
f\left(\left(1-t^{\rho}\right) a^{\rho}+t^{\rho} b^{\rho}\right) \leq \max \left\{f\left(a^{\rho}\right), f\left(b^{\rho}\right)\right\}
$$

By adding these inequalities we have

$$
\frac{1}{2}\left[f\left(t^{\rho} a^{\rho}+\left(1-t^{\rho}\right) b^{\rho}\right)+f\left(\left(1-t^{\rho}\right) a^{\rho}+t^{\rho} b^{\rho}\right)\right] \leq \max \left\{f\left(a^{\rho}\right), f\left(b^{\rho}\right)\right\}
$$

Multiplying both sides of (2.2) by $t^{\alpha \rho-1}$ and integrating the resulting inequality with respect to $t$ over $\left[a^{\rho}, b^{\rho}\right]$, we obtain

$$
\begin{aligned}
& \int_{0}^{1} t^{\alpha \rho-1} f\left(t^{\rho} a^{\rho}+\left(1-t^{\rho}\right) b^{\rho}\right) d t+\int_{0}^{1} t^{\alpha \rho-1} f\left(\left(1-t^{\rho}\right) a^{\rho}+t^{\rho} b^{\rho}\right) d t \\
= & \int_{a}^{b}\left(\frac{b^{\rho}-x^{\rho}}{b^{\rho}-a^{\rho}}\right)^{\alpha-1} f\left(x^{\rho}\right) \frac{x^{\rho-1}}{b^{\rho}-a^{\rho}} d x+\int_{a}^{b}\left(\frac{x^{\rho}-a^{\rho}}{b^{\rho}-a^{\rho}}\right)^{\alpha-1} f\left(x^{\rho}\right) \frac{x^{\rho-1}}{b^{\rho}-a^{\rho}} d x \\
= & \frac{1}{\left(b^{\rho}-a^{\rho}\right)^{\alpha}} \int_{a}^{b} \frac{x^{\rho-1}}{\left(b^{\rho}-x^{\rho}\right)^{1-\alpha}} f\left(x^{\rho}\right) d x+\frac{1}{\left(b^{\rho}-a^{\rho}\right)^{\alpha}} \int_{a}^{b} \frac{x^{\rho-1}}{\left(x^{\rho}-a^{\rho}\right)^{1-\alpha}} f\left(x^{\rho}\right) d x \\
= & \frac{\Gamma(\alpha)}{\rho^{1-\alpha}\left(b^{\rho}-a^{\rho}\right)^{\alpha}}\left[{ }^{\rho} \mathcal{I}_{a+}^{\alpha}(f \circ g)(b)+{ }^{\rho} \mathcal{I}_{b-}^{\alpha}(f \circ g)(a)\right] \\
\leq & \frac{2}{\rho \alpha} \max \left\{f\left(a^{\rho}\right), f\left(b^{\rho}\right)\right\}
\end{aligned}
$$

So we get desired result. The proof is completed.

Remark 2.1. In Theorem 2.1, taking limit $\rho \rightarrow 1$ we obtain inequality of (1.5).

Theorem 2.2. Let $\alpha>0$ and $\rho>0$. Let $f:\left[a^{\rho}, b^{\rho}\right] \rightarrow \mathbb{R}$ be a differentiable mapping on $\left[a^{\rho}, b^{\rho}\right]$ with $0 \leq a<b$. If $\left|f^{\prime}\right|$ is a quasi-convex function on $\left[a^{\rho}, b^{\rho}\right]$, then the following inequalities holds:

$$
\begin{aligned}
& \left|\frac{f\left(a^{\rho}\right)+f\left(b^{\rho}\right)}{2}-\frac{\alpha \rho^{\alpha} \Gamma(\alpha+1)}{2\left(b^{\rho}-a^{\rho}\right)^{\alpha}}\left[{ }^{\rho} \mathcal{I}_{a+}^{\alpha}(f \circ g)(b)+{ }^{\rho} \mathcal{I}_{b-}^{\alpha}(f \circ g)(a)\right]\right| \\
= & \frac{b^{\rho}-a^{\rho}}{\rho(\alpha+1)}\left(1-\frac{1}{2^{\rho(\alpha+1)}}\right) \max \left\{\left|f^{\prime}\left(a^{\rho}\right)\right|\left|f^{\prime}\left(b^{\rho}\right)\right|\right\}
\end{aligned}
$$

where $g(x)=x^{\rho}$. 
Proof. Using Lemma 1.2 and quasi-convex of $\left|f^{\prime}\right|$ with modulus, we get

$$
\begin{aligned}
& \left|\frac{f\left(a^{\rho}\right)+f\left(b^{\rho}\right)}{2}-\frac{\alpha \rho^{\alpha} \Gamma(\alpha+1)}{2\left(b^{\rho}-a^{\rho}\right)^{\alpha}}\left[{ }^{\rho} \mathcal{I}_{a+}^{\alpha}(f \circ g)(b)+{ }^{\rho} \mathcal{I}_{b-}^{\alpha}(f \circ g)(a)\right]\right| \\
\leq & \frac{b^{\rho}-a^{\rho}}{2} \int_{0}^{1}\left|\left(1-t^{\rho}\right)^{\alpha}-t^{\rho \alpha}\right| t^{\rho-1}\left|f^{\prime}\left(t^{\rho} a^{\rho}+\left(1-t^{\rho}\right) b^{\rho}\right)\right| d t \\
\leq & \frac{b^{\rho}-a^{\rho}}{2} \int_{0}^{1}\left|\left(1-t^{\rho}\right)^{\alpha}-t^{\rho \alpha}\right| t^{\rho-1} \max \left\{\left|f^{\prime}\left(a^{\rho}\right)\right|\left|f^{\prime}\left(b^{\rho}\right)\right|\right\} d t \\
= & \frac{b^{\rho}-a^{\rho}}{2} \max \left\{\left|f^{\prime}\left(a^{\rho}\right)\right|\left|f^{\prime}\left(b^{\rho}\right)\right|\right\} \\
& \times\left\{\int_{0}^{1 / 2^{1 / \rho}}\left[\left(1-t^{\rho}\right)^{\alpha}-t^{\rho \alpha}\right] t^{\rho-1} d t+\int_{1 / 2^{1 / \rho}}^{1}\left[t^{\rho \alpha}+\left(1-t^{\rho}\right)^{\alpha}\right] t^{\rho-1} d t\right\} \\
= & \frac{b^{\rho}-a^{\rho}}{\rho(\alpha+1)}\left(1-\frac{1}{2^{\alpha}}\right) \max \left\{\left|f^{\prime}\left(a^{\rho}\right)\right|\left|f^{\prime}\left(b^{\rho}\right)\right|\right\}
\end{aligned}
$$

where

$$
\begin{aligned}
& \int_{0}^{1 / 2^{1 / \rho}}\left[\left(1-t^{\rho}\right)^{\alpha}-t^{\rho \alpha}\right] t^{\rho-1} d t+\int_{1 / 2^{1 / \rho}}^{1}\left[t^{\rho \alpha}+\left(1-t^{\rho}\right)^{\alpha}\right] t^{\rho-1} d t \\
= & \frac{1}{\rho}\left\{\int_{0}^{1 / 2}\left[(1-u)^{\alpha}-u^{\alpha}\right] d u+\int_{1 / 2}^{1}\left[u^{\alpha}-(1-u)^{\alpha}\right] d u\right\} \\
= & \frac{2}{\rho(\alpha+1)}\left(1-\frac{1}{2^{\alpha}}\right) .
\end{aligned}
$$

The proof is completed.

Remark 2.2. In Theorem 2.2, taking limit $\rho \rightarrow 1$ we obtain inequality of (1.6).

Theorem 2.3. Let $\alpha>0$ and $\rho>0$. Let $f:\left[a^{\rho}, b^{\rho}\right] \rightarrow \mathbb{R}$ be a differentiable mapping on $\left[a^{\rho}, b^{\rho}\right]$ with $0 \leq a<b$. If $\left|f^{\prime}\right|^{q}$ is a quasi-convex function on $\left[a^{\rho}, b^{\rho}\right]$ and $s>1$, then the following inequalities holds:

$$
\begin{aligned}
& \left|\frac{f\left(a^{\rho}\right)+f\left(b^{\rho}\right)}{2}-\frac{\alpha \rho^{\alpha} \Gamma(\alpha+1)}{2\left(b^{\rho}-a^{\rho}\right)^{\alpha}}\left[{ }^{\rho} \mathcal{I}_{a+}^{\alpha}(f \circ g)(b)+{ }^{\rho} \mathcal{I}_{b-}^{\alpha}(f \circ g)(a)\right]\right| \\
= & \frac{b^{\rho}-a^{\rho}}{2}\left(\max \left\{\left|f^{\prime}\left(a^{\rho}\right)\right|^{q},\left|f^{\prime}\left(b^{\rho}\right)\right|^{q}\right\}\right)^{1 / q}\left(K_{1}+K_{2}\right)^{1 / s}
\end{aligned}
$$

where

$$
\begin{aligned}
K_{1} & =\frac{1}{\rho 2^{s+\frac{1-s}{\rho}}} B\left(s+\frac{1-s}{\rho}, \alpha s+1\right), \\
K_{2} & =\frac{\alpha s+1}{2 \rho}{ }_{2} F_{1}\left(1-s+\frac{s-1}{\rho}, 1 ; \alpha s+2 ; \frac{1}{2}\right),
\end{aligned}
$$

$\frac{1}{s}+\frac{1}{q}=1$ and $g(x)=x^{\rho}$ 
Proof. From Lemma 1.1, Lemma 1.2, Hölder inequality and quasi-convex of $\left|f^{\prime}\right|$ with proporties of modulus, we have

$$
\begin{aligned}
& \left|\frac{f\left(a^{\rho}\right)+f\left(b^{\rho}\right)}{2}-\frac{\alpha \rho^{\alpha} \Gamma(\alpha+1)}{2\left(b^{\rho}-a^{\rho}\right)^{\alpha}}\left[{ }^{\rho} \mathcal{I}_{a+}^{\alpha}(f \circ g)(b)+{ }^{\rho} \mathcal{I}_{b-}^{\alpha}(f \circ g)(a)\right]\right| \\
\leq & \frac{b^{\rho}-a^{\rho}}{2} \int_{0}^{1}\left|\left(1-t^{\rho}\right)^{\alpha}-t^{\rho \alpha}\right| t^{\rho-1}\left|f^{\prime}\left(t^{\rho} a^{\rho}+\left(1-t^{\rho}\right) b^{\rho}\right)\right| d t \\
\leq & \frac{b^{\rho}-a^{\rho}}{2}\left(\int_{0}^{1}\left|\left(1-t^{\rho}\right)^{\alpha}-t^{\rho \alpha}\right|^{s} t^{s(\rho-1)} d t\right)^{1 / s}\left(\int_{0}^{1}\left|f^{\prime}\left(t^{\rho} a^{\rho}+\left(1-t^{\rho}\right) b^{\rho}\right)\right|^{q} d t\right)^{1 / q} \\
\leq & \frac{b^{\rho}-a^{\rho}}{2}\left(\int_{0}^{1}\left|1-2 t^{\rho}\right|^{\alpha s} t^{s(\rho-1)} d t\right)^{1 / s}\left(\max \left\{\left|f^{\prime}\left(a^{\rho}\right)\right|^{q},\left|f^{\prime}\left(b^{\rho}\right)\right|^{q}\right\}\right)^{1 / q} \\
= & \frac{b^{\rho}-a^{\rho}}{2}\left(\max \left\{\left|f^{\prime}\left(a^{\rho}\right)\right|^{q},\left|f^{\prime}\left(b^{\rho}\right)\right|^{q}\right\}\right)^{1 / q} \\
& \times\left\{\int_{0}^{1 / 2^{1 / \rho}}\left(1-2 t^{\rho}\right)^{\alpha s} t^{s(\rho-1)} d t+\int_{1 / 2^{1 / \rho}}^{1}\left(2 t^{\rho}-1\right)^{\alpha s} t^{s(\rho-1)} d t\right\}^{1 / s} \\
= & \frac{b^{\rho}-a^{\rho}}{2}\left(\max \left\{\left|f^{\prime}\left(a^{\rho}\right)\right|^{q},\left|f^{\prime}\left(b^{\rho}\right)\right|^{q}\right\}\right)^{1 / q}\left(K_{1}+K_{2}\right)^{1 / s}
\end{aligned}
$$

where

$$
\begin{aligned}
K_{1}=\int_{0}^{1 / 2^{1 / \rho}}\left(1-2 t^{\rho}\right)^{\alpha s} t^{s(\rho-1)} d t & =\frac{1}{\rho 2^{s+\frac{1-s}{\rho}}} \int_{0}^{1} u^{s-1+\frac{1-s}{\rho}}(1-u)^{\alpha s} d u \\
& =\frac{1}{\rho 2^{s+\frac{1-s}{\rho}}} B\left(s+\frac{1-s}{\rho}, \alpha s+1\right) \\
K_{2}=\int_{1 / 2^{1 / \rho}}^{1}\left(2 t^{\rho}-1\right)^{\alpha s} t^{s(\rho-1)} d t & =\frac{1}{2^{s+\frac{1-s}{\rho}} \rho} \int_{0}^{1} u^{\alpha s}(1+u)^{s-1+\frac{1-s}{\rho}} d u \\
& =\frac{\alpha s+1}{2 \rho}{ }_{2} F_{1}\left(1-s+\frac{s-1}{\rho}, 1 ; \alpha s+2 ; \frac{1}{2}\right)
\end{aligned}
$$

So, if we use (2.6), (2.7) in (2.5), we obtain desired result.

Remark 2.3. In Theorem 2.3, taking limit $\rho \rightarrow 1$ we obtain inequality of (1.7).

Theorem 2.4. Let $\alpha>0$ and $\rho>0$. Let $f:\left[a^{\rho}, b^{\rho}\right] \rightarrow \mathbb{R}$ be a differentiable mapping on $\left[a^{\rho}, b^{\rho}\right]$ with $0 \leq a<b$. If $\left|f^{\prime}\right|^{q}$ is a quasi-convex function on $\left[a^{\rho}, b^{\rho}\right]$ and $q \geq 1$, then the following inequalities holds:

$$
\begin{aligned}
& \left|\frac{f\left(a^{\rho}\right)+f\left(b^{\rho}\right)}{2}-\frac{\alpha \rho^{\alpha} \Gamma(\alpha+1)}{2\left(b^{\rho}-a^{\rho}\right)^{\alpha}}\left[{ }^{\rho} \mathcal{I}_{a+}^{\alpha}(f \circ g)(b)+{ }^{\rho} \mathcal{I}_{b-}^{\alpha}(f \circ g)(a)\right]\right| \\
\leq & \frac{b^{\rho}-a^{\rho}}{\rho(\alpha+1)}\left(1-\frac{1}{2^{\alpha}}\right)\left(\max \left\{\left|f^{\prime}\left(a^{\rho}\right)\right|^{q},\left|f^{\prime}\left(b^{\rho}\right)\right|{ }^{q}\right\}\right)^{1 / q}
\end{aligned}
$$

where $g(x)=x^{\rho}$. 
Proof. From Lemma 1.2, quasi-convex of $\left|f^{\prime}\right|$ and using power-mean inequality with proporties of modulus, we have

$$
\begin{aligned}
& \left|\frac{f\left(a^{\rho}\right)+f\left(b^{\rho}\right)}{2}-\frac{\alpha \rho^{\alpha} \Gamma(\alpha+1)}{2\left(b^{\rho}-a^{\rho}\right)^{\alpha}}\left[{ }^{\rho} \mathcal{I}_{a+}^{\alpha}(f \circ g)(b)+{ }^{\rho} \mathcal{I}_{b-}^{\alpha}(f \circ g)(a)\right]\right| \\
\leq & \frac{b^{\rho}-a^{\rho}}{2} \int_{0}^{1}\left|\left(1-t^{\rho}\right)^{\alpha}-t^{\rho \alpha}\right| t^{\rho-1}\left|f^{\prime}\left(t^{\rho} a^{\rho}+\left(1-t^{\rho}\right) b^{\rho}\right)\right| d t \\
\leq & \frac{b^{\rho}-a^{\rho}}{2}\left(\int_{0}^{1}\left|\left(1-t^{\rho}\right)^{\alpha}-t^{\rho \alpha}\right| t^{\rho-1} d t\right)^{1-1 / q} \\
& \times\left(\int_{0}^{1}\left|\left(1-t^{\rho}\right)^{\alpha}-t^{\rho \alpha}\right| t^{\rho-1}\left|f^{\prime}\left(t^{\rho} a^{\rho}+\left(1-t^{\rho}\right) b^{\rho}\right)\right|^{q} d t\right)^{1 / q} \\
\leq & \frac{b^{\rho}-a^{\rho}}{2}\left(\int_{0}^{1}\left|\left(1-t^{\rho}\right)^{\alpha}-t^{\rho \alpha}\right| t^{\rho-1} d t\right)^{1-1 / q} \\
& \times\left(\max \left\{\left|f^{\prime}\left(a^{\rho}\right)\right|^{q},\left|f^{\prime}\left(b^{\rho}\right)\right|^{q}\right\}\right)^{1 / q}\left(\int_{0}^{1}\left|\left(1-t^{\rho}\right)^{\alpha}-t^{\rho \alpha}\right| t^{\rho-1} d t\right)^{1 / q} \\
= & \frac{b^{\rho}-a^{\rho}}{2}\left(\int_{0}^{1}\left|\left(1-t^{\rho}\right)^{\alpha}-t^{\rho \alpha}\right| t^{\rho-1} d t\right)\left(\max \left\{\left|f^{\prime}\left(a^{\rho}\right)\right|^{q},\left|f^{\prime}\left(b^{\rho}\right)\right|^{q}\right\}\right)^{1 / q}
\end{aligned}
$$

Using (2.4) we get desired result.

Remark 2.4. In Theorem 2.4, taking limit $\rho \rightarrow 1$ we obtain inequality of (1.8).

\section{Acknowledgement}

This research is supported by Ordu University Scientific Research Projects Coordination Unit (BAP). Project Number: YKD-17224

\section{REFERENCES}

[1] M.U. Awan, M.A. Noor, M.V. Mihai and K.I. Noor, Fractional Hermite-Hadamard inequalities for differentiablesGodunova-Levin functions, Filomat, 30(12) (2016), 3235C-3241.

[2] H. Chen, U.N. Katugampola, Hermite-Hadamard and Hermite-Hadamard-Fejer type inequalities for generalized fractional integrals, J. Math. Anal. Appl., 446, 1274-1291.

[3] S.S. Dragomir and C.E.M. Pearce, Selected Topics on Hermite-Hadamard Inequalities and Applications, RGMIA Monographs, Victoria University, 2000.

[4] D.A. Ion, Some estimates on the Hermite-Hadamard inequality through quasi-convex functions, Ann. Univ. Craiova, Math. Comp. Sci. Ser., 34 (2007), 82-87.

[5] U.N. Katugampola, New approach to generalized fractional derivatives, Bull. Math. Anal. Appl., 6(4), (2014), 1-15.

[6] A.A. Kilbas, H.M. Srivastava, J.J. Trujillo, Theory and applications of fractional differential equations, Amsterdam: Elsevier; (2006).

[7] M. A. Noor, K. I. Noor, and M. U. Awan, Generalized convexity and integral inequalities, Appl. Math. Inform. Sci., 9(1) (2015),233-243.

[8] M. E. Özdemir, Ç. Yıldız, The Hadamards inequality for quasi-convex functions via fractional integrals, Annals of the University of Craiova, Ann. Univ. Craiova, Math. Comp. Sci. Series, 40(2) (2013), 167-173. 
[9] A.P. Prudnikov, Y.A. Brychkov, O.I. Marichev, Integral and series. In: Elementary Functions, vol. 1. Nauka, Moscow; (1981).

[10] E.D. Rainville, Special Functions, The Mcmillan Company, New York, 1960.

[11] M. Z. Sarıkaya, E. Set, H. Yaldız, N. Başak, Hermite-Hadamard's inequalities for fractional integrals and related fractional inequalities, Math. Comput. Model., 57 (2013), 2403-2407.

[12] E. Set, M.Z. Sarikaya, M.E. Özdemir, H. Yildirim, The Hermite-Hadamards inequality for some convex functions via fractional integrals and related results, J. Appl. Math. Stat. Inf., 10(2) (2014), 69-83.

[13] E. Set, İ. İşcan, F. Zehir, On some new inequalities of Hermite-Hadamard type involving harmonically convex functions via fractional integrals, Konuralp J. Math., 3(1) (2015), 42-55.

[14] E. Set, A.O. Akdemir, I. Mumcu, Hermite-Hadamard's Inequality and its Extensions for Conformable Fractional Integrals of Any Order $\alpha>0$, ResearchGate, https://www.researchgate.net/publication/303382221.

[15] E. Set, B. Çelik, Fractional Hermite-Hadamard type inequalities for quasi-convex functions, Ordu Univ. J. Sci. Tech., 6(1) (2016), 137-149.

[16] E. Set, A.O. Akdemir, B. Çelik, On Generalization of Fejer Type Inequalities via fractional integral operator, ResearchGate, https://www.researchgate.net/publication/311452467.

[17] E. Set, A.O. Akdemir, I. Mumcu, Ostrowski type inequalities involving special functions via conformable fractional integrals, J. Adv. Math. Stud., 10(3) (2017), 386-395.

[18] E. Set, M.A. Noor, M.U. Awan, A. Gözpinar, Generalized Hermite-Hadamard type inequalities involving fractional integral operators, J. Ineq. Appl., 2017(169) (2017), 1-10.

[19] E. Set,J. Choi, A. Gözpinar, Hermite-Hadamard type inequalities for the generalized k-fractional integral operators, J. Ineq. Appl., 2017 (2017), Art. ID 206.

[20] E. Set, M.E. Özdemir, M.Z. Sarıkaya, Inequalities of Hermite-Hadamard type for functions whose derivatives absolute values are m-convex, AIP Conf. Proc., 1309(1) (2010), 861-863.

[21] H.M. Srivastava and J. Choi, Zeta and $q$-Zeta Functions and Associated Series and Integrals, Elsevier Science Publishers, Amsterdam, London and New York, 2012. 